secret message in the form of a committed bit - the value of which is kept hidden until a designated time.

Bit commitment is potentially useful for voting and other applications in which privacy is key, but ultrasecure quantum commitment schemes have proved difficult to implement.

Felix Bussières of the University of Geneva in Switzerland and his colleagues experimentally demonstrated a protocol proposed in 2012 for keeping committed bits secret. The technique, which relies on the constancy of the speed of light, can prevent insecure communication between remote locations for periods of up to 15 milliseconds, perhaps long enough to enable high-speed stock trading.

Phys. Rev. Lett. 111, 180504 (2013)

\section{REGENERATIVE MEDICINE}

\section{Embryo protein has healing power}

A protein that is present mainly in developing embryos gives some adult tissues an exceptional ability to heal.

The protein Lin28a

controls growth and development in animals from worms to humans, but it is rarely found in adult tissues in vertebrates.

When researchers led by George Daley at Boston Children's Hospital in Massachusetts engineered mice so that the protein was produced into adulthood, the animals' hair grew faster and puncture wounds in their ears healed more thoroughly. Newborn animals were more likely to regrow the tips of severed digits.

Lin28a represses an antigrowth regulatory program and boosts the production of many metabolic enzymes. In doing so, the researchers say, the protein induces a state that enhances tissue repair. Cell 155, 778-792 (2013) For a longer story on this research, see go.nature.com/8cejq3
INFLAMMATORY DISEASE

\section{Sun synchronizes immune system}

Daily light cycles help to set animals' response to infection.

Lora Hooper of the

University of Texas

Southwestern Medical Center in Dallas and her colleagues showed that a protein that controls whether certain white blood cells mature is in turn controlled by proteins that set daily rhythms. When mice were exposed to abnormal light cycles, rhythm-setting proteins sent signals to the immune system, prompting it to make more inflammatory cells in the gut.

Inflammation protected the mice against bacterial infection, but it also made them more susceptible to inflammatory bowel disease and colitis. The findings might explain why shift workers and others with disrupted sleep cycles are more prone to immune disorders. Science 342, 727-730 (2013)

\section{SEISMOLOGY}

\section{Injected gas makes Earth rumble}

Earthquakes that shook an old Texas oil field over the past few years could be due to injections of carbon dioxide gas. Previous tremors in the area were tied to wastewater injections, but these are the first events attributed to forcing pressurized gas underground.

Two years after significant gas injections began in the oil field to boost production

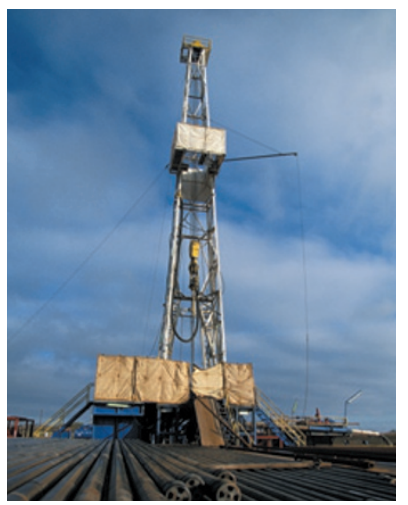

COMMUNITY CHOICE

The most viewed papers in science

VIROLOGY

\title{
New flu virus found in bats
}

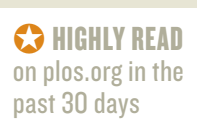

The flat-faced fruit bat (Artibeus planirostris; pictured) in Peru carries a flu virus that was previously unknown to science.

$\mathrm{H} 18 \mathrm{~N} 11$ is the second influenza A virus to be identified in bats, and both were found by a team led by Ruben Donis of the Centers for Disease Control and Prevention in Atlanta, Georgia. The team found huge genetic diversity in bat influenza A viruses: four of eight bat viral gene segments harboured more genetic variation than did corresponding viral segments in other mammals and

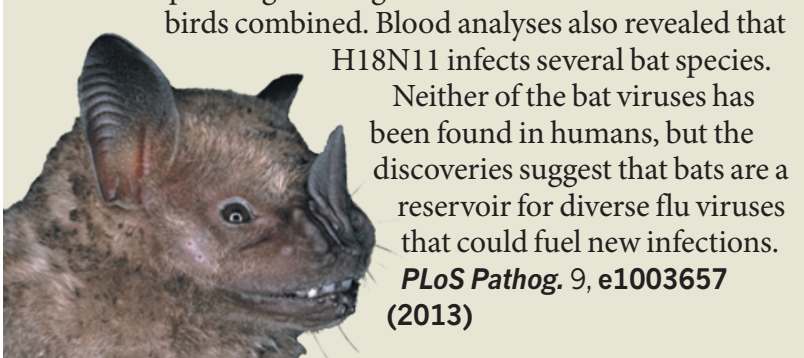

(pictured), the area experienced a spate of small earthquakes, 18 of which reached magnitudes between 3 and 4.4. Wei Gan and Cliff Frohlich at the University of Texas at Austin have now determined the precise location and timing of the quakes.

The locations correlate with gas injection sites and suggest that slippage occurred along a previously unidentified fault. Nearby fields where gas was also injected did not show such seismic activity, however, and the researchers say that more data are needed to predict where gas injection could trigger tremors.

Proc. Natl Acad. Sci. USA http://doi.org/pxs (2013)

For a longer story on this research, see go.nature.com/x4hhn4

\section{MEDICAL MICROBIOLOGY}

\section{Gut microbes linked to arthritis}

Unfriendly bacteria in the gut might bring on inflammation in the joints.

Dan Littman at the New York University School of
Medicine and his colleagues collected stool samples from people with arthritis. Sequencing experiments on gut bacteria showed that individuals with newly diagnosed arthritis were more likely to harbour the bacterium Prevotella copri than were individuals without the condition.

High levels of $P$. copri and related species correlated with low levels of beneficial microbes that are thought to suppress the immune system and metabolize vitamins into forms that are absorbed by the bloodstream. When inoculated with P. copri, mice became more sensitive to colitis, and the levels of beneficial microbes in their guts dropped.

Gut bacteria have long been implicated in arthritis, but this is the first time that researchers have correlated the onset of human disease with a particular microbial species. eLife 2, e01202 (2013)

\section{$\rightarrow$ NATURE.COM}

For the latest research published by Naturevisit:

www.nature.com/latestresearch 